\title{
The Cosmological Constant and Pioneer Anomaly from Weyl Spacetimes and Mach's Principle
}

\author{
Carlos Castro \\ Center for Theoretical Studies of Physical Systems \\ Clark Atlanta University, Atlanta, GA. 30314, castro@ctps.cau.edu
}

January 2009, Revised March 2009

\begin{abstract}
It is shown how Weyl's geometry and Mach's Holographic principle furnishes both the magnitude and sign (towards the sun) of the Pioneer anomalous acceleration $a_{P} \sim-c^{2} / R_{\text {Hubble }}$ firstly observed by Anderson et al. Weyl's Geometry can account for both the origins and the value of the observed vacuum energy density (dark energy). The source of dark energy is just the dilaton-like Jordan-Brans-Dicke scalar field that is required to implement Weyl invariance of the most simple of all possible actions. A nonvanishing value of the vacuum energy density of the order of $10^{-123} M_{\text {Planck }}^{4}$ is found consistent with observations. Weyl's geometry accounts also for the phantom scalar field in modern Cosmology in a very natural fashion.
\end{abstract}

Keywords: Dark Energy, Weyl Geometry, Brans-Dicke-Jordan Gravity, Pioneer Anomaly, Cosmology.

The problem of dark energy is one of the most challenging problems facing Cosmology today with a vast numerable proposals for its solution, we refer to the recent monograph [1], [3] and references therein. In [4] we have shown how Weyl's geometry (and its scaling symmetry) is instrumental to solve this dark energy riddle. In this letter we will show how Weyl's geometry in an elegant fashion can account for both the magnitude and sign of the Pioneer anomalous acceleration [5]. Before starting we must emphasize that our procedure [4] was quite different than previous proposals [2] to explain dark matter ( instead of dark energy ) in terms of Brans-Dicke gravity. It is not only necessary to include the Jordan-Brans-Dicke scalar field $\phi$ but it is essential to have a Weyl geometric extension and generalization of Riemannian geometry ( ordinary gravity ).

Weyl's geometry main feature is that the norm of vectors under parallel infinitesimal displacement going from $x^{\mu}$ to $x^{\mu}+d x^{\mu}$ change as follows 
$\delta\|V\| \sim\|V\| A_{\mu} d x^{\mu}$ where $A_{\mu}$ is the Weyl gauge field of scale calibrations that behaves as a connection under Weyl transformations :

$$
A_{\mu}^{\prime}=A_{\mu}-\partial_{\mu} \Omega(x) . \quad g_{\mu \nu} \rightarrow e^{2 \Omega} g_{\mu \nu} .
$$

involving the Weyl scaling parameter $\Omega\left(x^{\mu}\right)$. The Weyl covariant derivative operator acting on a tensor $\mathbf{T}$ is defined by $D_{\mu} \mathbf{T}=\left(\nabla_{\mu}+\omega(\mathbf{T}) A_{\mu}\right) \mathbf{T}$; where $\omega(\mathbf{T})$ is the Weyl weight of the tensor $\mathbf{T}$ and the derivative operator $\nabla_{\mu}=\partial_{\mu}+\Gamma_{\mu}$ involves a connection $\Gamma_{\mu}$ which is comprised of the ordinary Christoffel symbols $\left\{\rho_{\mu \nu}^{\rho}\right\}$ plus the $A_{\mu}$ terms

$$
\Gamma_{\mu \nu}^{\rho}=\left\{\begin{array}{l}
\rho \\
\mu \nu
\end{array}\right\}+\delta_{\mu}^{\rho} A_{\nu}+\delta_{\nu}^{\rho} A_{\mu}-g_{\mu \nu} g^{\rho \sigma} A_{\sigma}
$$

The Weyl gauge covariant operator $\partial_{\mu}+\Gamma_{\mu}+w(\mathbf{T}) A_{\mu}$ obeys the condition

$$
D_{\mu}\left(g_{\nu \rho}\right)=\nabla_{\mu}\left(g_{\nu \rho}\right)+2 A_{\mu} g_{\nu \rho}=0 .
$$

where $\nabla_{\mu}\left(g_{\nu \rho}\right)=-2 A_{\mu} g_{\nu \rho}=Q_{\mu \nu \rho}$ is the non-metricity tensor. Torsion can be added [17] if one wishes but for the time being we refrained from doing so. The connection $\Gamma_{\mu \nu}^{\rho}$ is Weyl invariant so that the geodesic equation in Weyl spacetimes is Weyl-covariant under Weyl gauge transformations (scalings)

$$
\begin{gathered}
d s \rightarrow e^{\Omega} d s ; \frac{d x^{\mu}}{d s} \rightarrow e^{-\Omega} \frac{d x^{\mu}}{d s} ; \frac{d^{2} x^{\mu}}{d s^{2}} \rightarrow e^{-2 \Omega}\left[\frac{d^{2} x^{\mu}}{d s^{2}}-\frac{d x^{\mu}}{d s} \frac{d x^{\nu}}{d s} \partial_{\nu} \Omega\right] . \\
g_{\mu \nu} \rightarrow e^{2 \Omega} g_{\mu \nu} ; A_{\mu} \rightarrow A_{\mu}-\partial_{\mu} \Omega ; \quad A^{\mu} \rightarrow e^{-2 \Omega}\left(A^{\mu}-\partial^{\mu} \Omega\right) ; \quad \Gamma_{\mu \nu}^{\rho} \rightarrow \Gamma_{\mu \nu}^{\rho} .
\end{gathered}
$$

thus, the Weyl covariant geodesic equation transforms under Weyl scalings as

$$
\begin{gathered}
\frac{d^{2} x^{\rho}}{d s^{2}}+\Gamma_{\mu \nu}^{\rho} \frac{d x^{\mu}}{d s} \frac{d x^{\nu}}{d s}-A_{\mu} \frac{d x^{\mu}}{d s} \frac{d x^{\rho}}{d s}=0 \rightarrow \\
e^{-2 \Omega}\left[\frac{d^{2} x^{\rho}}{d s^{2}}+\Gamma_{\mu \nu}^{\rho} \frac{d x^{\mu}}{d s} \frac{d x^{\nu}}{d s}-A_{\mu} \frac{d x^{\mu}}{d s} \frac{d x^{\rho}}{d s}\right]=0 .
\end{gathered}
$$

The Weyl weight of the metric $g_{\nu \rho}$ is 2 . The meaning of $D_{\mu}\left(g_{\nu \rho}\right)=0$ is that the angle formed by two vectors remains the same under parallel transport despite that their lengths may change. This also occurs in conformal mappings of the complex plane. The Weyl covariant derivative acting on a scalar $\phi$ of Weyl weight $\omega(\phi)=-1$ is defined by

$$
D_{\mu} \phi=\partial_{\mu} \phi+\omega(\phi) A_{\mu} \phi=\partial_{\mu} \phi-A_{\mu} \phi .
$$

The Weyl scalar curvature in $D$ dimensions and signature $(-,+,+,+\ldots$.$) is { }^{1}$

$$
\mathcal{R}_{W e y l}=R_{\text {Riemann }}-(D-1)(D-2) A_{\mu} A^{\mu}-2(D-1) \nabla_{\mu} A^{\mu} .
$$

\footnotetext{
${ }^{1}$ Some authors define their $A_{\mu}$ field with the opposite sign as $-A_{\mu}$ which changes the sign in the last term of the Weyl scalar curvature (7)
} 
Having introduced the basics of Weyl geometry our starting action is the Weylinvariant Jordan-Brans-Dicke-like action involving the scalar $\phi$ field and the scalar Weyl curvature $\mathcal{R}_{W e y l}$

$$
\begin{gathered}
S\left[g_{\mu \nu}, A_{\mu}, \phi\right]=S\left[g_{\mu \nu}^{\prime}, A_{\mu}^{\prime}, \phi^{\prime}\right] \Rightarrow \\
\frac{1}{16 \pi} \int d^{4} x \sqrt{|g|}\left[\phi^{2} \mathcal{R}_{W e y l}\left(g_{\mu \nu}, A_{\mu}\right)-\frac{1}{2} g^{\mu \nu}\left(D_{\mu} \phi\right)\left(D_{\nu} \phi\right)-V(\phi)\right]= \\
\frac{1}{16 \pi} \int d^{4} x \sqrt{\left|g^{\prime}\right|}\left[\left(\phi^{\prime}\right)^{2} \mathcal{R}_{W \text { eyl }}^{\prime}\left(g_{\mu \nu}^{\prime}, A_{\mu}^{\prime}\right)-\frac{1}{2} g^{\prime \mu \nu}\left(D_{\mu}^{\prime} \phi^{\prime}\right)\left(D_{\nu}^{\prime} \phi^{\prime}\right)-V\left(\phi^{\prime}\right)\right]
\end{gathered}
$$

where under Wey scalings one has

$$
\begin{gathered}
\phi^{\prime}=e^{-\Omega} \phi ; \quad g_{\mu \nu}^{\prime}=e^{2 \Omega} g_{\mu \nu} ; \quad \mathcal{R}_{W e y l}^{\prime}=e^{-2 \Omega} \mathcal{R}_{W e y l} ; \quad V\left(\phi^{\prime}\right)=e^{-4 \Omega} V(\phi) \\
\sqrt{\left|g^{\prime}\right|}=e^{4 \Omega} \sqrt{|g|} ; \quad D_{\mu}^{\prime} \phi^{\prime}=e^{-\Omega} D_{\mu} \phi ; \quad A_{\mu}^{\prime}=A_{\mu}-\partial_{\mu} \Omega
\end{gathered}
$$

The effective Newtonian coupling $G$ is defined as $\phi^{-2}=G(\phi)$, it is spacetime dependent in general and has a Weyl weight equal to 2. Despite that one has not introduced any explicit dynamics to the $A_{\mu}$ field (there are no $F_{\mu \nu} F^{\mu \nu}$ terms in the action (9)) one still has the constraint equation obtained from the variation of the action w.r.t to the $A^{\mu}$ field and which leads to the pure-gauge configurations provided $\phi \neq 0$

$$
\begin{gathered}
\frac{\delta S}{\delta A^{\mu}}=0 \Rightarrow 6\left(2 A_{\mu} \phi^{2}-\partial_{\mu}\left(\phi^{2}\right)\right)+\frac{1}{2}\left(2 A_{\mu} \phi^{2}-\partial_{\mu}(\phi)^{2}\right)= \\
-\left(6+\frac{1}{2}\right) D_{\mu} \phi^{2}=-2\left(6+\frac{1}{2}\right) \phi D_{\mu} \phi=0 \Rightarrow A_{\mu}=\partial_{\mu} \log (\phi) .
\end{gathered}
$$

Hence, a variation of the action w.r.t the $A_{\mu}$ field leads to the pure gauge solutions (10) which is tantamount to saying that the scalar $\phi$ is Weyl-covariantly constant $D_{\mu}=0$ in any gauge $D_{\mu} \phi=0 \rightarrow e^{-\Omega} D_{\mu} \phi=D_{\mu}^{\prime} \phi^{\prime}=0$ (for nonsingular gauge functions $\Omega \neq \pm \infty$ ). Therefore, the scalar $\phi$ does not have true local dynamical degrees of freedom from the Weyl spacetime perspective. Since the gauge field is a total derivative, under a local gauge transformation with gauge function $\Omega=\log \phi$, one can gauge away (locally) the gauge field and have $A_{\mu}^{\prime}=0$ in the new gauge. Globally, however, this may not be the case because there may be topological obstructions. Therefore, the last constraint equation (10) in the gauge $A_{\mu}^{\prime}=0$, forces $\partial_{\mu} \phi^{\prime}=0 \Rightarrow \phi^{\prime}=\phi_{o}=$ constant. Consequently $G^{\prime}=\phi^{\prime-2}$ is also constrained to a constant $G_{N}$ and one may set $G_{N} \phi_{o}^{2}=1$, where $G_{N}$ is the observed Newtonian constant today.

The pure-gauge configurations leads to the Weyl integrability condition $F_{\mu \nu}=$ $\partial_{\mu} A_{\nu}-\partial_{\nu} A_{\mu}=0$ when $A_{\mu}=\partial_{\mu} \Omega$, and means physically that if we parallel transport a vector under a closed loop, as we come back to the starting point, the norm of the vector has not changed; i.e, the rate at which a clock ticks does not change after being transported along a closed loop back to the initial 
point; and if we transport a clock from $A$ to $B$ along different paths, the clocks will tick at the same rate upon arrival at the same point $B$. This will ensure, for example, that the observed spectral lines of identical atoms will not change when the atoms arrive at the laboratory after taking different paths ( histories ) from their coincident starting point. If $F_{\mu \nu} \neq 0$ the Weyl geometry is no longer integrable.

With the Weyl-invariant action (9) at hand one may find a realization of dark energy (the observed cosmological constant ) as it was shown in [4] and the Pioneer anomaly (as we will show next) by choosing two different gauges. The cosmological gauge $A_{\mu}$ in spherical coordinates is defined by

$$
A_{r}=-\frac{1}{R_{\text {Hubble }}} ; A_{t}=A_{\varphi}=A_{\theta}=0 .
$$

and is associated with the present day Hubble scale $R_{\text {Hubble }} \sim 10^{28} \mathrm{~cm}$. The other gauge is the Einstein gauge

$$
A_{\mu}^{\prime}=0=A_{\mu}-\partial_{\mu} \Omega \Rightarrow A_{r}=-\frac{1}{R_{H}}=\partial_{r} \Omega \Rightarrow \Omega=-\frac{r}{R_{H}} .
$$

From eq- (10) we learned that

$$
A_{\mu}=\partial_{\mu} \log \phi \Rightarrow A_{r}=-\frac{1}{R_{H}} \Rightarrow \phi=e^{-r / R_{H}} \phi_{o} .
$$

such that the Newtonian couplings in the two different gauges "scale-frames of reference" are related as follows

$$
\frac{\phi^{2}}{\phi_{o}^{2}}=\frac{G_{N}}{G(\phi)} \Rightarrow G(\phi)=G_{N} e^{2 r / R_{H}}
$$

the effective Newtonian coupling in the cosmological gauge (cosmological "scaleframe of reference" ) increases with distance : it has an anti-screening effect which is also compatible with the findings in the asymptotic safety scenario of Quantum Gravity [11] and the lattice approach to Quantum Gravity as well $[12]$.

In the Einstein gauge $A_{\mu}^{\prime}=0$, using the Weyl covariant constraint of eq(10) stating that the scalar field $\phi$ is Weyl-covariantly constant (without true dynamics) and for non-singular gauge functions $\Omega \neq \pm \infty$, one can deduce that

$$
D_{\mu}^{\prime} \phi^{\prime}=\partial_{\mu} \phi^{\prime}-A_{\mu}^{\prime} \phi^{\prime}=\partial_{\mu} \phi^{\prime}=e^{-\Omega} D_{\mu} \phi=0 \Rightarrow \phi^{\prime}=\phi_{o} .
$$

Hence, the action (9) in the gauge $A_{\mu}^{\prime}=0 \Leftrightarrow \phi^{\prime}=\phi_{o}=$ constant becomes

$$
\frac{1}{16 \pi} \int d^{4} x \sqrt{\left|g^{\prime}\right|}\left[\left(\phi_{o}\right)^{2} R_{\text {Riemann }}\left(g_{\mu \nu}^{\prime}\right)-V\left(\phi_{o}\right)\right]
$$

which is just the ordinary Einstein-Hilbert action with a cosmological constant $\Lambda$ given by $2 \Lambda \equiv G_{N} V\left(\phi_{o}\right)$ because $\phi_{o}^{2}=1 / G_{N}$. The equations of motion associated with the action (15) are 


$$
R_{\mu \nu}^{\prime}-\frac{1}{2} g_{\mu \nu}^{\prime} R^{\prime}+\Lambda g_{\mu \nu}^{\prime}=0
$$

and which admit the static spherically symmetric solutions corresponding to (Anti) de Sitter-Schwarzschild metrics

$d s^{2}=-\left(1-\frac{2 G_{N} M}{r}-\frac{\Lambda}{3} r^{2}\right) d t^{2}+\left(1-\frac{2 G_{N} M}{r}-\frac{\Lambda}{3} r^{2}\right)^{-1} d r^{2}+r^{2}\left(\sin ^{2} \theta d \varphi^{2}+d \theta^{2}\right)$.

It is known that de Sitter-Schwarzschild metric solutions (17) for very large values of $r$ (discarding the $2 G_{N} M / r$ terms) leads to the static spherically symmetric reparametrized version of the standard temporal dependent de Sitter metrics $d s^{2}=-d t^{2}+R_{H}^{2} \cosh ^{2}\left(t / R_{H}\right)\left(d \Omega_{3}\right)^{2}(c=1)$, after a judicious change of coordinates is performed where $\Omega_{3}$ is the 3 -dim solid angle associated with the 3 -sphere $S^{3}$.

The metric solutions in the cosmological gauge $A_{r}=-\frac{1}{R_{H}}$ are simply obtained by a conformal transformation

$g_{\mu \nu}=e^{-2 \Omega} g_{\mu \nu}^{\prime} \Rightarrow g_{t t}=e^{-2 \Omega} g_{t t}^{\prime}=-e^{2 r / R_{H}}\left(1-\frac{2 G_{N} M}{r}-\frac{\Lambda}{3} r^{2}\right)$, etc $\ldots$.

The conformal scalings of the (Anti) de Sitter-Schwarzschild metrics are solutions to equations of motion based on the action $S\left[g_{\mu \nu}, A_{\mu}, \phi\right]$ as a direct consequence of the Weyl symmetry invariance of the action : the equations of motion in one gauge are related to the equations of motion in another gauge by a simple gauge transformation (scaling) of the field variables. Hence, a conformal scaling of the equations of motion (16) yields the following equations of motion in terms of the new variables $g_{\mu \nu}, A_{\mu}, \phi$, (with $\left.A_{\mu}=\partial_{\mu} \log \phi\right)$

$$
G_{\mu \nu}=R_{\mu \nu}-\frac{1}{2} g_{\mu \nu} R=\frac{8 \pi T_{\mu \nu}^{\text {matter }}}{\phi^{2}}+T_{\mu \nu}(\phi)
$$

where in our case $T_{\mu \nu}^{\text {matter }}=-\frac{2}{\sqrt{|g|}} \frac{\delta S_{\text {matter }}}{\delta g^{\mu \nu}}=0$. The effective stress energy tensor $T_{\mu \nu}(\phi)$ is

$$
\begin{gathered}
T_{\mu \nu}(\phi)=-\frac{4}{\phi^{2}}\left(\nabla_{\mu} \phi\right)\left(\nabla_{\nu} \phi\right)+\frac{1}{\phi^{2}} g_{\mu \nu}\left(\nabla^{\rho} \phi\right)\left(\nabla_{\rho} \phi\right)+ \\
\frac{2}{\phi}\left(\nabla_{\mu} \nabla_{\nu} \phi\right)-\frac{2}{\phi} g_{\mu \nu}\left(\nabla_{\rho} \nabla^{\rho} \phi\right)-g_{\mu \nu} \frac{V(\phi)}{2 \phi^{2}} .
\end{gathered}
$$

The $\nabla_{\mu}$ are the ordinary covariant derivatives involving solely the Levi-Civita connection (without $A_{\mu}$ terms) and $R_{\mu \nu}, R$ are the Riemannian Ricci curvature tensor and Ricci curvature scalar, respectively. The effective stress energy tensor $T_{\mu \nu}(\phi)$ turns out to coincide precisely with the effective stress energy tensor of a Brans-Dicke-Jordan (BDJ) scalar field $\Phi=\phi^{2}$ whose $\omega$ parameter is $\omega=-3 / 2$ and $U\left(\Phi=\phi^{2}\right)=V(\phi)$ given by 


$$
\begin{gathered}
T_{\mu \nu}^{B D J}\left(\Phi=\phi^{2} ; \omega=-\frac{3}{2}\right)=-\frac{3}{2 \phi^{4}}\left[\left(\nabla_{\mu} \phi^{2}\right)\left(\nabla_{\nu} \phi^{2}\right)-\frac{1}{2} g_{\mu \nu}\left(\nabla^{\rho} \phi^{2}\right)\left(\nabla_{\rho} \phi^{2}\right)\right]+ \\
\frac{1}{\phi^{2}}\left[\left(\nabla_{\mu} \nabla_{\nu} \phi^{2}\right)-g_{\mu \nu}\left(\nabla_{\rho} \nabla^{\rho} \phi^{2}\right)\right]-g_{\mu \nu} \frac{U\left(\phi^{2}\right)}{2 \phi^{2}}
\end{gathered}
$$

One recognizes that $\omega=-3 / 2$ is the critical value of the $\omega$ parameter in BDJ scalar theories of gravity [6] based on the action

$$
S^{B D J}(\Phi ; \omega)=\frac{1}{16 \pi} \int d^{4} x \sqrt{|g|}\left[\Phi R_{\text {Riemann }}-\frac{\omega}{\Phi} g^{\mu \nu}\left(\nabla_{\mu} \Phi\right)\left(\nabla_{\nu} \Phi\right)-U(\Phi)\right] .
$$

whose $g_{\mu \nu}$ field equations coincide with $(19,20)$ when $\omega=-3 / 2$ and $\Phi=\phi^{2}$. A variation of $S^{B D J}(\Phi ; \omega)$ w.r.t the BDJ scalar $\Phi=\phi^{2}$, and after taking the trace of the field equations $(19,20)$ in order to eliminate the Riemannian curvature scalar $R$ yields after some algebra the following

$$
\nabla_{\mu} \nabla^{\mu} \Phi=\frac{1}{2 \omega+3}\left[\Phi \frac{d U(\Phi)}{d \Phi}-2 U(\Phi)\right] .
$$

when $\omega=-3 / 2 \Rightarrow 2 \omega+3=0$ the denominator of (23) becomes zero leading to an unacceptable singularity in (23) unless the numerator is also zero which then determines uniquely the nontrivial potential to be $U(\Phi)=\lambda \Phi^{2}=\lambda \phi^{4}$. Performing a Weyl scaling of the latter potential and recurring to the scaling relations (9) yields the constant vacuum energy density $\lambda e^{-4 \Omega} \phi^{4}=\lambda \phi_{o}^{4}=V_{o}$. In the case of an expanding de Sitter universe with $H_{o}=1 / R_{H}(c=1)$ where $R_{H}$ is the Hubble radius related to the throat size of the 4-dim hyperboloid of constant scalar curvature $\left(R=12 / R_{H}^{2}\right)$ embedded in flat pseudo-Euclidean 5-dim space, one has $\Lambda \equiv 3 H_{o}^{2}=3 / R_{H}^{2}$ so that

$$
\begin{gathered}
2 \Lambda=\frac{6}{R_{H}^{2}}=G_{N} V_{o}=16 \pi G_{N} \rho \Rightarrow \\
\rho=\frac{3}{8 \pi} \frac{1}{G_{N} R_{H}^{2}}=\frac{3}{8 \pi}\left(\frac{L_{P}}{R_{H}}\right)^{2} M_{\text {Planck }}^{4} \sim 10^{-123} M_{\text {Planck }}^{4} .
\end{gathered}
$$

and which is the correct value for the observed vacuum energy density after identifying $\phi_{o}^{-2}=G_{N}=L_{P}^{2}$ and $M_{\text {Planck }}=L_{P}^{-1}$ in terms of the Planck scale $L_{P}$ (in natural $\hbar=c=1$ units) and setting the present-day Hubble radius to be of the order $R_{H} \sim 10^{61} L_{P}$. A Jordan-Brans-Dicke gravity model within the context of ordinary Riemannian geometry with the parameter $\omega=-\frac{3}{2}$ yields the observed vacuum energy density (cosmological constant) to very high precision because the temporal flow of the scalar field $\phi(t)$ in ordinary Riemannian geometry, from $t=0$ to $t=t_{o}$ (today), has the same numerical effects (as far as the vacuum energy density is concerned) as if there were Weyl scalings from the variable field configuration $\phi(t)$ to the constant field configuration $\phi_{o}$. Hence, Weyl scalings in Weyl geometry can recapture the flow of time which is consistent with Segal's Conformal Cosmology [14], in such a fashion that an 
expanding universe may be visualized as Weyl scalings of a static universe. This temporal flow of the vacuum energy density, from very high values in the past, to very small values today, is not a numerical coincidence but is the signal of an underlying Weyl geometry (conformal invariance) operating in cosmology, combined with the dynamics of a Brans-Dicke-Jordan scalar field.

The value of the dimensionless parameter $\lambda$ is then $\lambda=6 G_{N} H_{o}^{2}=\left(6 H_{o}^{2} / \phi_{o}^{2}\right)$ so that the potential can be rewritten as $U\left(\Phi=\phi^{2}\right)=\left(6 H_{o}^{2} \phi_{o}^{2}\right)\left(\phi / \phi_{o}\right)^{4}$. By scaling $\phi$ to the constant $\phi_{o}$ one recovers then $V_{o}=6 H_{o}^{2} \phi_{o}^{2}$ as expected. The key point that we wish to advance here is that the observed vacuum energy density (24) is consistent with the critical value of the BDJ parameter $\omega=-3 / 2$ determined from the Weyl scaling symmetry properties of the action (8) and that uniquely fixes the nontrivial potential to be of the form $U(\Phi)=\lambda \Phi^{2}=\lambda \phi^{4}$. A variable potential that will afterwards scale to the constant $V_{o} \neq 0$ value related to the observed vacuum energy density via the relations in (24) because Weyl's symmetry allows us to set $\phi_{o}^{-2}=G_{N}=L_{P}^{2}$. A trivial potential $U\left(\phi^{2}\right)=0$ would have lead to the trivial solution $V_{o}=0$ yielding a zero vacuum energy density.

Above we have pointed out that the scalar $\phi$ does not have true local dynamical degrees of freedom from the Weyl spacetime perspective as a result of the Weyl-covariant constancy condition (9). However from the Riemannian point of view one can see by inspection from the BDJ action (22) when $\omega=-3 / 2$ and $\Phi=\phi^{2}$ that the term $6\left(\nabla_{\mu} \phi\right)\left(\nabla^{\mu} \phi\right)$ has the wrong sign for a kinetic term when the signature is $(-,+,+,+)$ and behaves then like a phantom-scalar field in modern Cosmology. However this is not a problem because from the Weyl spacetime perspective the scalar $\phi$ is devoid of true dynamics $D_{\mu} \phi=0$ and behaves like Dirac's gauge function scalar field [17] : it is a compensator scalar field required to implement Weyl invariance in the action (8). Therefore, Weyl's geometry accounts for the phantom scalar field in a very natural fashion.

After this discussion we turn finally to the Pioneer anomaly. Upon expanding the exponential conformal factor of (18) in a power series yields

$$
\begin{gathered}
-g_{t t}=\left(1+\frac{2 r}{R_{H}}+\frac{1}{2}\left(\frac{2 r}{R_{H}}\right)^{2}+\ldots\right)\left(1-\frac{2 G_{N} M}{r}-\frac{\Lambda}{3} r^{2}\right)= \\
1-\frac{2 G_{N} M}{r}-\frac{\Lambda}{3} r^{2}+\frac{2 r}{R_{H}}-\frac{4 G_{N} M}{R_{H}}-\frac{2 \Lambda r^{3}}{3 R_{H}}+\ldots \ldots . .
\end{gathered}
$$

For scales $r<<R_{H}$ corresponding to the Pioneer-Sun's distance one may neglect the higher order corrections in the expansion. From the $g_{t t}$ component one can read-off the corrections to the Newtonian potential in natural units $c=1$ from the Newtonian limit of Einstein's gravity : $-g_{t t} \sim 1+2 V$ leading to

$$
V_{\text {effective }}(r)=-\frac{G_{N} M}{r}-\frac{\Lambda}{6} r^{2}+\frac{r}{R_{H}}-\frac{2 G_{N} M}{R_{H}}-\frac{\Lambda r^{3}}{3 R_{H}}+\ldots .
$$

Therefore the acceleration (radial force per unit mass) acting on the Pioneer 
spacecraft after reinserting the speed of light $c$ in its proper units and by setting $\Lambda=3 / R_{H}^{2}$ is given by

$$
\frac{F_{r}}{m}=a=-\frac{\partial V_{e f f}}{\partial r}=-\frac{G_{N} M}{r^{2}}-\frac{c^{2}}{R_{H}}\left(1-\frac{r}{r_{H}}-3\left(\frac{r}{R_{H}}\right)^{2}\right)+\ldots
$$

Concluding : one recovers the correct order of magnitude and sign (pointing towards the sun) of the Pioneer anomalous acceleration $a_{P}=-c^{2} / R_{H}=$ $-8.98 \times 10^{-8} \mathrm{~cm} / \mathrm{sec}^{2}$ which is given exactly by the first term of the corrections to the Newtonian (radial) acceleration $\left(-\frac{G_{N} M}{r^{2}}\right)$ in eq- $(27)$. The experimental value [5] of the magnitude is $\left|a_{P}\right|=(8.74 \pm 1.33) \times 10^{-8} \mathrm{~cm} / \mathrm{sec}^{2}$. The other terms of eq-(27) correspond to the contribution of the cosmological constant and further corrections stemming from the conformal factor in eq-(18).

One can also explain the Pioneer anomaly from the Weyl covariant geodesic equation (5). In the Einstein gauge $A_{\mu}^{\prime}=0$ one recovers the ordinary geodesic in Riemannian spacetime with the (Anti ) de Sitter-Schwarzschild (AdSS) metric $g_{\mu \nu}^{\prime}$ of eq-(17); however in another gauge $A_{\mu} \neq 0, g_{\mu \nu}=e^{2 r / R_{H}} g_{\mu \nu}^{A d S S}$ the additional contributions to the connection $\Gamma_{\mu \nu}^{\rho}$ in the geodesic equation (5) (stemming from the $A_{\mu}$ terms in eq-(2)) would appear from the Riemannian geometry perspective as if there were an extra "force" (a pseudo force, a "fifth force" ) involving terms of the form $\left(g^{\mu \nu}-V^{\mu} V^{\nu}\right) A_{\nu}$ (where $A_{\nu}=\partial_{\nu} \log (\phi)$ ) acting on a test particle and endowing it with an (apparent) non-geodesic motion. In the non-relativistic Newtonian limit, for purely radial motions with values of $r$ such $R_{H}>>r>>2 G_{N} M ; d s \sim c d t ;\left(V^{r}\right)^{2} \sim \frac{v^{2}}{c^{2}}$, the term $\left(g^{r r}-\right.$ $\left.V^{r} V^{r}\right) A_{r} \sim-\frac{1}{R_{H}}$. The extra contribution to the radial acceleration becomes $\Delta\left(\frac{d^{2} x^{r}}{d s^{2}}\right) \sim \Delta\left(\frac{1}{c^{2}} \frac{d^{2} x^{r}}{d t^{2}}\right)=-\frac{1}{R_{H}}$ so that $\Delta\left(\frac{d^{2} x^{r}}{d t^{2}}\right) \sim-\frac{c^{2}}{R_{H}}$ as expected. Notice however that this anomalous acceleration (non-geodesic motion in Riemannian spacetime) is just a gauge artifact because from the Weyl spacetime geometry perspective the test particle always follows a geodesic, prior and after a gauge transformation is performed since the geodesic equation (5) is covariant under Weyl scalings.

Let us try to answer the poignant question posed by many : Why planets revolving around the sun in elliptical orbits $d o n^{\prime} t$ experience such anomalous acceleration?. Since the planets are bound to the solar system they are not moving freely along the hyperbolas (geodesics) of the 4-dim hyperboloid represented by the asymptotically de Sitter space. The geodesics (hyperbolic paths) from the point of view of the de Sitter metric are not "straight lines" (geodesics) from the point of view a flat Minkowski metric, and hence, this could be the physical meaning of the anomalous Pioneer acceleration w.r.t the sun (solar system). The sun (solar system) is not a truly inertial system within the background de Sitter space perspective. The solar system can be seen as a non-expanding penny on an expanding balloon (de Sitter universe). Since the path traced by the Pioneer spacecraft is indeed a hyperbola [5], one can view the Pioneer spacecraft after it has left the outer edge of the solar system as it were a free particle (devoid of external forces ) moving on a 4-dim hyperboloid de Sitter space, because the present universe is in an accelerated expansion de Sitter phase. 
This is not the end of the story. Firstly, one must also invoke Mach's principle within the context of BDJ scalar theories of gravity: the effective spacetime dependent Newtonian coupling $G(\phi)=\phi^{-2}$ is determined by the distribution of masses of the universe. The authors [9] have implemented a precise formulation of Mach's principle (the so-called "Mach's Holographic Principle") where inertial frames can be fully determined by the metric if one specifies the bulk stress energy tensor plus boundary conditions, and where the latter can be replaced (mimic) by the appropriate boundary stress energy on a hypersurface. This boundary matter plays the same role of the distant stars for bulk observers and selects a frame with respect to which inertial and accelerated motion have a meaning. Secondly, one can view this boundary as a hypersurface separating an interior region (the solar system) where Weyl's symmetry is broken from an exterior region where Weyl's symmetry is preserved. A Gauss-Mainardi-Codazzi (GMC) formalism has been used by [10] to facilitate the study of thin shells of matter in gravity. It allows the possibility of sewing together two spacetime regions with different conformal properties. This GMC boundary-value problem in Weyl spacetime has been studied [10] to show how to construct an interior domain (the solar system in our case, an atom in their case ) where Weyl invariance is broken, while in the exterior region Weyl invariance is fully preserved. Therefore, the interior domain corresponds to our solar system (where no anomaly occurs), while the exterior geometry (where the Pioneer spacecraft is located) is conformally invariant and is subjected to the anomalous acceleration. Thirdly, the boundary matter separating these two regions can be physically realized by the Edgeworth-Kuiper main-belt stretching from roughly 30 to 55 AU (Astronomical Units) and which is a region of the Solar System beyond the planets [21], while the scattered objects of the belt reach past Uranus at approximately $20 \mathrm{AU}^{2}$.

One may extend Weyl's scaling symmetry to the full-fledged conformal group $S O(4,2)$ by adding the conformal boost generators [8] and by constructing $S O(4,2)$ invariant actions. Conformal boosts bestow a massive particle with a constant acceleration, whereas ordinary momentum transformations amount to translations. This explains why there is an interplay between large cosmological scales ( Hubble radius $R_{H}$ ) and small microscopic scales ( Planck scale $\left.L_{P}\right)$ in the value of the observed vacuum energy density $\rho=\frac{3}{8 \pi} L_{P}^{-2} R_{H}^{-2}$ in eq-(24) : it stems from the fact that the canonical conjugate variable to the conformal-boost-momentum generator is the inverse of the position coordinate $z^{\mu}=\frac{x^{\mu}}{x_{\mu} x^{\mu}}$ [8]. Since under inversions large and small scales are interchanged this explains the interplay between large and small scales in the value of the vacuum energy density $\rho(24)$ and the expression for $a_{P}=-\frac{c^{2}}{R_{H}}$.

Boundary conditions are crucial when one integrates by parts and may not allow discarding total derivative terms in the action. Nontrivial topologies like wormholes $\mathbf{R} \times S^{1} \times S^{2}$, toruses $\mathbf{R} \times S^{1} \times S^{1} \times S^{1}$ and topological defects like cosmic strings and Nielsen-Olesen vortices will furnish different results as well

\footnotetext{
${ }^{2}$ We thank Frank (Tony) Smith for pointing out the relevance of Uranus orbit in the physical measurements by Anderson et al [5]
} 
because one may not be able to gauge to zero globally a pure gauge configuration $A_{\mu}=\partial_{\mu} \log (\phi)$ found in eq-(10). A thorough discussion of these effects and the breaking of Weyl scaling symmetry can be found in [7]. The role of dilatation symmetry in higher dimensions and the vanishing of the cosmological constant from the Renormalization Group perspective has been studied by [19]. Weylconformally invariant light-like p-brane Theories have been constructed by [20]. For a current monograph on Wey's geometry and its applications in Physics see [16]. Furthermore, one should work with most general Lagrangian involving dynamics for $A_{\mu}$ and adding spinorial matter fields, etc...

$\mathcal{L}=\phi^{2} \mathcal{R}_{W e y l}\left(g_{\mu \nu}, A_{\mu}\right)-\frac{1}{4} F_{\mu \nu} F^{\mu \nu}-\frac{1}{2} g^{\mu \nu}\left(D_{\mu} \phi\right)\left(D_{\nu} \phi\right)-V(\phi)+L_{\text {matter }}+\ldots .$.

The $L_{\text {matter }}$ must involve the full fledged Weyl gauge covariant derivatives acting on scalar and spinor fields .

Inflationary solutions in Weyl spacetimes based in a pure-gauge ansatz were investigated long ago by Kao [18]. The inflationary solution by Kao required a very large cosmological constant, it was argued how this very large value of the cosmological constant during the inflationary period could be diluted to the observed very small value as the universe expanded by a 60 e-fold factor $e^{60}$ during a very short time interval of the order of $10^{-35}$ seconds. In this respect, Kao's results agree with ours. The vacuum energy problem from the Finsler Geometry perspective has been analyzed by [13]. The Pioneer anomaly has been analyzed within the context of scalar-tensor and nonsymmetric metric theories of Gravity by [15] but it does not involve Weyl's geometry and requires fitting the observed data by tuning the values of two extra parameters.

A different study of the Pioneer anomaly based on the Weyl geometry associated with the temporal dependent Friedmann-Robertson-Walker (FRW) metrics was made by [14]. Our results differ from [14] in several key aspects. Firstly, the field equations in [14] were not correct because of the wrongly variation of the term $\sqrt{|g|} g^{\mu \nu} \delta\left(\phi^{2} \mathcal{R}_{\mu \nu}\right)$ in the action. Only when $\phi$ is constant such variation is a total derivative and can be dropped from the field equations. When the BDJ scalar field $\phi$ is spacetime dependent, it is the variation of this term which leads precisely to the extra components of the effective stress energy tensor in eqs- $(20,21)$. Secondly, we have found in the $A_{\mu}=0$ gauge, to begin with, (Anti) de Sitter-Schwarzchild solutions such that for large $r>>2 G_{N} M$ correspond to the static spherically symmetric reparametrization of the temporal-dependent ( Anti ) de Sitter solutions (17) and which belong to a special family of FRW metrics whose scaling function is $a(t)=\cosh \left(t / R_{H}\right)$. While in the $A_{r}=-1 / R_{H}$ gauge we have conformally rescaled (Anti) de Sitter-Schwarzchild solutions (18). These results differ from [14] because his metric solution in the $A_{\mu}=0$ gauge is just the flat Minkowski spacetime metric, while his metric in the $A_{t}=H(t)$ gauge is of the FRW type with scaling factor $a(t)=e^{\int H(t) d t}$. Thirdly, the anomalous acceleration in [14] had for magnitude the value $a=H_{O} v_{\text {Pioneer }}=\frac{c}{R_{H}} v_{\text {Pioneer }}<<\frac{c^{2}}{R_{H}}$. This former value is 
several orders of magnitude smaller ${ }^{3}$ than the experimental value observed by Anderson et al [5]. The expression in (27) furnishes the correct magnitude and sign of the anomalous acceleration.

Other more conventional approaches to the Pioneer anomaly and to the flyby anomaly can be found in the websites [22]. In particular, differential heating may account for as much as $1 / 3$ of the observed acceleration (see in [22] the article by David Harris). If there are other plausible explanations involving conventional physics these would not necessarily falsify our results here unless there are strong arguments against integrable Weyl geometry ${ }^{4}$.

\section{Acknowledgements}

We thank Frank (Tony) Smith for discussions; M. Bowers for assistance and to the referee for his important suggestions to improve this work.

\section{References}

[1] S. Weinberg, Cosmology (Oxford University Press, 2008).

[2] H. Kim, "Can the Brans-Dicke gravity possibly with $\Lambda$ be a theory of Dark matter ? "astro-ph/0604055. M. Arik and M. Calik, "Can Brans-Dicke scalar field account for dark energy and dark matter ? gr-qc/0505035. Chao-Jun Feng, "Ricci Dark Energy in Brans-Dicke Theory" arXiv : 0806.0673.

[3] S. Capozzielo, V. Cardone and A. Troisi, "Jour. of Cosmology and Astroparticle Physics 08 (2006) 001. S. Capozziello, S. Nojiri and S. Odintsov, Phys. Letts B 634 (2006) 93.

[4] C. Castro, "How Weyl Geometry solves the Riddle of Dark Energy" in Quantization Astrophysics, Brownian Motion and Supersymmetry pp. 8896 (eds F. Smarandache and V. Christianato, Math. Tiger, Chennai, India 2007). Foundations of Physics vol 37, no. 3 (2007) 366." On Dark Energy, Weyl Geometry and Brans-Dicke-Jordan Scalar Field" to appear in the Global Journal of Science and Technology (New Dehli, 2009 ). Mod. Phys. Lett A17 (2002) 2095. Mod. Phys. Lett A 21, no. 35 (2006) 2685.

[5] J. Anderson, P. Laing, E. Lau, A. Liu, M. Nieto and S. Turyshev Phys.Rev.Lett. 81 (1998) 2858; Mod.Phys.Lett. A17 (2002).

[6] V. Faraoni, Cosmology in Scalar-Tensor Gravity (Kluwer Academic Publishers, 2003). Y. Fujii and K. Maeda, The Scalar-Tensor Theory of Gravitation (Cambridge University Press 2003).

\footnotetext{
${ }^{3}$ This problem persists in the most recent work by Scholz arXiv : 0805.2557 despite correcting the variation $\sqrt{|g|} g^{\mu \nu} \delta\left(\phi^{2} \mathcal{R}_{\mu \nu}\right)$

${ }^{4}$ We thank the referee for raising this poignant question
} 
[7] F. Hehl, J. McCrea, E. Mielke and Y. Ne'eman, Phys. Reports 258 (1995) 1 .

[8] R. Aldrovandi, J. Beltran Almeida and J. Pereira, J. Geom. Phys 56 (2006) 1042. C. Castro, Adv. Studies in Theor. Phys. 2, no. 7 (2008) 309-332

[9] J. Khoury and M. Parikh, "Mach's Holographic Principle" arXiv : hepth/0612117.

[10] W. Wood and G. Papini, Phys. Rev. D 45, no. 10 (1992) 3617.

[11] M. Reuter, Proceedings of the 2008 Conference on Quantum Gravity, Challenges and Perspectives Bad Honneff ( H. Nicolai et al, eds ).

[12] H. Hamber, " Quantum Gravity on the Lattice " arXiv : 0901.0964.

[13] H. Brandt, J. Modern Optics 51, 2753-2759 (2004).

[14] E. Scholz, " On the Geometry of Cosmological Model Building" grqc/0511113

[15] J. Moffat, " Modified Gravitational Theory and the Pioneer 10 and 11 Spacecraft Anomalous Acceleration" gr-qc/0405076.

[16] R. Carroll, Fluctuations, information, gravity and the Quantum Potential, (Springer Verlag 2006). "Remarks on Conformal Mass and Quantum Mass" Progress in Physics vol 2 (April) (2008) 89-90. "On the quantum potential" Arima Publ, 2007.

[17] M. Israelit, Found. Phys 29, 1303 (1999). Found. Phys 32, 295 (2002). Found. Phys 32, 945 (2002). The Weyl-Dirac theory and our universe (Nova Science Publishers 1999).

[18] W. Kao, Phys. Letts A 149 (1990) 76.

[19] C. Wetterich, "Dilatation symmetry in higher dimensions and the vanishing of the cosmological constant" arXiv : 0806.0741.

[20] E. Guendelman and A. Kaganovich, Phys. Rev D 53, 7020 (1996). Phys. Rev D 60, 065004 (1999). Int. J. Mod. Phys A 17, 417 (2002). E. Guendelman, A. Kaganovich, E. Nissimov and S. Pacheva, "Weyl-conformally invariant light-like p-brane Theories" [arXiv.org: hep-th/0409078].

[21] http://en.wikipedia.org/wiki/Kuiper_belt

[22] http://en.wikipedia.org/wiki/Pioneer_anomaly; http://en.wikipedia.org/wiki/Flyby_anomaly 